\title{
Mutations in PIEZO2 contribute to Gordon syndrome, Marden-Walker syndrome and distal arthrogryposis: A bioinformatics analysis of mechanisms
}

\author{
YANBO MA $^{1}$, YANTAO ZHAO ${ }^{2}$, ZHEN CAI $^{1}$ and XIUYAN HAO ${ }^{1}$ \\ ${ }^{1}$ Department of Stomatology, Linyi People's Hospital, Linyi, Shandong 276000; \\ ${ }^{2}$ Department of Stomatology, Luozhuang Central Hospital, Linyi, Shandong 276017, P.R. China
}

Received January 5, 2018; Accepted May 17, 2018

DOI: $10.3892 /$ etm.2019.7381

\begin{abstract}
Piezo type mechanosensitive ion channel component 2 (PIEZO2) is a mechanically activated ion channel. Mutations in PIEZO2 may cause distal arthrogryposis 3 (DA3)/Gordon syndrome (GS), DA5, Marden-Walker syndrome (MWS) and associated diseases. To date, no systematic study has analyzed and compared the influence of different gene mutations of PIEZO2 on its transcription, as well as translation and protein function. Therefore, the objective of the present study was to systematically assess the effect of different pathological mutations of PIEZO2 on transcription, translation, as well as protein structure and function that contribute to GS/DA3, DA5, MWS and associated diseases based on a bioinformatics analysis using the Pubmed, ClinVar, RaptorX and Phyre 2 online databases. The results indicated the presence of 27 pathological mutations in PIEZO2, including dominant and recessive mutations. Dominant mutations were mainly located in the C-terminal region, whereas recessive mutations were mainly localized in the $\mathrm{N}$-terminal region, and most reported mutation sites exhibited high evolutionary conservation among different species. Loss-of-function mutations result in nonsense-mediated transcript decay or premature termination codons, consequently leading to a lack of PIEZO2 protein, whereas gain-of-function mutations may lead to increased PIEZO2-associated channel activity. The bioinformatics analysis results also indicated that the p.Ala1486Pro, p.Thr2221Ile and p.Glu2727del mutations modify the secondary structure of the PIEZO2 protein, while p.Thr2221Ile, p.Arg2718Leu and p.Arg2718Pro mutations reduce the solvent accessibility of PIEZO2 protein. Furthermore, the p.Ala1486Pro,
\end{abstract}

Correspondence to: Ms Xiuyan Hao, Department of Stomatology, Linyi People's Hospital, 27 East Jiefang Road, Linyi, Shandong 276000, P.R. China

E-mail: lyhaoxiuyan@163.com

Key words: mutations, Piezo type mechanosensitive ion channel component 2, Gordon syndrome, Marden-Walker syndrome, bioinformatic analysis
p.Thr2221Ile, p.Ser2223Leu, p.Thr2356Met, p.Arg2686His, p.Arg2718Leu, p.Arg2718Pro and p.Glu2727del mutations affect the transmembrane region. These changes of PIEZO2 may contribute to a gain-of-function of PIEZO2. Variable clinical phenotypes were present between and among the gainand loss-of-function mutations linked with PIEZO2-associated disease, which implied that different mutations in PIEZO2 have different pathophysiological effects. Of course, further functional studies to explore the precise structure and function of PIEZO2 are necessary and may offer useful clues for the prevention and treatment of associated diseases.

\section{Introduction}

Distal arthrogryposis 3 (DA3)/Gordon syndrome (GS), DA5 and Marden-Walker syndrome (MWS) share a broad spectrum of similar phenotypes to describe congenital contractures of multiple joints that mainly involve congenital contractures of hands and feet, cleft palate, ptosis, cerebellar malformations, ophthalmoplegia, as well as pulmonary hypertension, which may be caused by decreased intrauterine movement, or due to neurological, muscle or connective tissue development disease (1-8). Previous studies also indicated that mechanotransduction is important for these biological and pathological processes, including sensory perception and embryonic development of organs, which is mediated by mechanosensation in proprioceptors, including muscle spindles in or the Golgi tendon organs in tendons that are able to sense mechanical forces upon cell membranes through mechanically activated ion channels, and propagate proprioceptive information by different nerve fibers (9).

Piezo type mechanosensitive ion channels (PIEZOs), including PIEZO component 1 (PIEZO1) and PIEZO2, are very large proteins with numerous predicted transmembrane domains per subunit, and are evolutionarily conserved in plants and animals (10). PIEZOs are expressed in a broad range of different tissue and cell types, including urinary bladder, lungs, kidneys, cartilage and dorsal root ganglion (DRGs) (11). In 2010, PIEZO1 and -2 were identified as the mechanically activated ion channels, and to have crucial roles in numerous mechanotransduction processes, including touch perception, proprioception and vascular development (12). A 
previous study indicated that constitutive deletion of PIEZO1 and -2 in mice leads to developmental lethality (13). PIEZO1 has a crucial role in the development of the mouse vasculature, and is required for mechanical force-induced cation influx in red blood cells $(14,15)$. PIEZO2 is expressed in a subset of DRG neurons that innervate the skin, hair follicles and Merkel cells to form low-threshold mechanoreceptors for the detection of light touch in mammals $(13,16-18)$. PIEZO2 is also a principal mechanically activated mechanotransducer in low-threshold skeletal muscle-innervating proprioceptors in mice (17).

Mutations in PIEZO2 have been reported to cause DA3, DA5 and MWS. Numerous dominant gain-of-function mutations, as well as recessive loss-of-function mutations, have been reported, including gain-of-function mutations that destabilize inactivation structures and lead to an overall increase of calcium influx, and frameshift mutations and out-of-frame exon skipping that lead to termination of protein synthesis through premature termination codons or nonsense-mediated decay of PIEZO2 transcripts (2-7). In spite of the marked improvement achieved by previous studies, a systematic study analyzing and comparing the influence of different mutations on PIEZO2 transcription, translation and protein function is currently lacking, to the best of our knowledge. Therefore, the present study aimed to systematically evaluate the effect of different pathological mutations of PIEZO2 on its transcription, as well as on translation and protein structure/function that contribute to DA3, DA5, MWS and associated diseases based on a bioinformatics analysis.

\section{Materials and methods}

Acquisition of pathological mutation information of PIEZO2. The pathological mutation information for PIEZO2 was obtained from the ClinVar database (https://www.ncbi.nlm. nih.gov/clinvar/), and further information was yielded from published studies found on PubMed. A number of studies that reported exact mutations in PIEZO2 (1-8) were included in the present study. However, certain studies that reported pathological deletions or repetitions in the chromosome region that included PIEZO2, but did not exactly confirm whether PIEZO2 was the pathological gene were excluded from the present study (19-21).

Conservation analysis of mutation sites in PIEZO2. The amino acid sequences of human PIEZO2 protein, as well as Monopterus albus, Mus musculus, Odocoileus virginianus texanus, Pogona vitticeps and Seriola dumerili PIEZO2 protein, were obtained from the National Center for Biotechnology Information (NCBI) website (https://www. ncbi.nlm.nih.gov/protein/). The amino acid sequence was saved in FASTA format, and the conservation of mutation sites in PIEZO2 was compared between different species using BioEdit software (version 7.0.5; downloaded from http://www. mbio.ncsu.edu/bioedit/bioedit.html).

Effect of gain-of-function mutations of PIEZO2 on protein structure. In the RaptorX database (http://raptorx.uchicago. edu/StructurePropertyPred/predict/) $(22,23)$ and the Phyre2 database (http://www.sbg.bio.ic.ac.uk/phyre2/html/page. cgi?id=index) (24), the amino acid sequences of normal and mutant PIEZO proteins were entered and the protein structures were predicted and analyzed.

\section{Results}

Reported mutation information of PIEZO2. A total of 27 pathological mutations were obtained from the ClinVar database and references, which included 6 de novo mutations, 10 dominant mutations and 11 recessive mutations. Among these mutations, gain-of-function mutations (dominant/de novo mutations) were located in the middle and C-terminal region, but mainly in the C-terminal region, particularly in the 52nd exon region. Loss-of-function mutations (recessive mutations) were located in the middle and $\mathrm{N}$-terminal region, but mainly in the $\mathrm{N}$-terminal region (Table I).

Conservation of mutation sites in PIEZO2. The sequence blast results of the PIEZO2 protein obtained from BioEdit software (version 7.0.5; downloaded from http://www.mbio. ncsu.edu/bioedit/bioedit.html) indicated that most mutation sites exhibited high conservation among different species, particularly in the C-terminal region. For mammals, all mutation sites exhibited high conservation in Homo sapiens, Mus musculus and Odocoileus virginianus texanus. However, the p.R462* mutation was distinctive between humans and other species (Table II).

Effect of gain-of-function mutations of PIEZO2 on protein structure and function. Regarding recessive mutations of PIEZO2, a previous study has indicated that they lead to loss-of-function of PIEZO2 due to nonsense-mediated decay of PIEZO2 transcripts or termination of protein synthesis through premature termination codons (3).

Regarding gain-of-function mutations, including E2727del and I802F, a previous study indicated that these two abovementioned mutations of PIEZO2 facilitate faster recovery of mechanically activated currents from inactivation, with E2727del leading to a slowing of inactivation, resulting in increased channel activity in response to mechanical stimulus (6). However, how these mutations influence the structure of PIEZO2 protein to affect the protein function remains to be elucidated. In the present study, the bioinformatical analysis results indicated that these mutations, including p.Ala1486Pro, p.Thr2221Ile and p.Glu2727del, modify the $\alpha$-helix structure, whereas p.Thr2356Met would modify the $\alpha$-helix and transmembrane helix structure (Fig. 1). In addition, the p.Thr2221Ile, p.Arg2718Leu and p.Arg2718Pro mutations lead to a reduction of the solvent accessibility of the PIEZO2 protein, whereas p.Arg2718Pro may change the $\alpha$-helix to a loop structure for the 8 -state secondary structure (SS8), and modifies the $\alpha$-helix to a coil for SS3 (Fig. 2).

The p.Ala1486Pro mutation changes the 32 nd transmembrane region (S32) of PIEZO2 protein, while the p.Thr2221Ile and p.Ser2223Leu mutations influence S32-S37 of the PIEZO2 protein. p.Thr2356Met changes S37. p.Arg2686His, p.Arg2718Leu, p.Arg2718Pro and p.Glu2727del modify S39 (Fig. 3). 
Table I. Pathological mutation information of PIEZO2.

\begin{tabular}{|c|c|c|c|c|c|c|}
\hline Author/year & $\begin{array}{c}\text { Gene } \\
\text { variation }\end{array}$ & Exon & $\begin{array}{l}\text { Protein } \\
\text { variation }\end{array}$ & $\begin{array}{c}\text { Disease/ } \\
\text { phenotypes }\end{array}$ & Inheritance & (Refs.) \\
\hline McMillin MJ, et al 2014 & $\begin{array}{l}\text { NM_022068.3:c.8238_8245 } \\
\text { delGACTAGAG }\end{array}$ & 52 & p.Trp2746Terfs ${ }^{a}$ & GS & De novo & $(5)$ \\
\hline McMillin MJ, et al 2014 & NM_022068.3:c.8215T>C & 52 & p.Ser2739Pro & DA5 & Dominant & $(5)$ \\
\hline McMillin MJ, et al 2014 & NM_022068.3:c.8208delA & 52 & p.Tyr2737Ilefs & DA5 & De novo & $(5)$ \\
\hline McMillin MJ, et al 2014 & $\begin{array}{l}\text { NM_022068.3:c. } \\
\text { 8181_8183delAGA }\end{array}$ & 52 & p.Glu2727del & DA5 & Dominant/de novo & $(5)$ \\
\hline Coste B, et al 2013 & NM_022068.2:c.8179_8181del & 52 & p.Glu2727del & DA5 & Dominant & (6) \\
\hline McMillin MJ, et al 2014 & NM_022068.3:c.8153G>C & 52 & p.Arg2718Pro & DA5 & De novo & $(5)$ \\
\hline McMillin MJ, et al 2014 & NM_022068.3:c.8153G>T & 52 & p.Arg2718Leu & DA5 & Dominant & $(5)$ \\
\hline $\begin{array}{l}\text { McMillin MJ, et al 2014; } \\
\text { Alisch F, et al } 2017\end{array}$ & NM_022068.3:c.8057G>A & 52 & p.Arg2686His & DA5, GS & Dominant/de novo & $(2,5)$ \\
\hline McMillin MJ, et al 2014 & NM_022068.3:c.8056C>T & 52 & p.Arg2686Cys & MWS & De novo & $(5)$ \\
\hline McMillin MJ, et al 2014 & NM_022068.3:c.7067C>T & 45 & p.Thr2356Met & DA5 & Dominant & $(5)$ \\
\hline McMillin MJ, et al 2014 & NM_022068.3:c.6668C>T & 45 & p.Ser2223Leu & DA5 & De novo & $(5)$ \\
\hline McMillin MJ, et al 2014 & NM_022068.3:c.6662C>T & 43 & p.Thr2221Ile & DA5 & Dominant/de novo & $(5)$ \\
\hline a & NM_022068.3:c.5895G>A & 38 & p.Trp1965Ter & $\mathrm{b}$ & Recessive & \\
\hline Delle Vedove A, et al 2016 & NM_022068.3:c.5621delT & 37 & p.Leu1874Argfs & c & Recessive & (3) \\
\hline Chesler AT, et al 2016 & NM_022068.3:c.5054G>C & 35 & p.Arg1685Pro & d & Recessive & $(7)$ \\
\hline Chesler AT, et al 2016 & NM_022068.3:c.5053C>T & 35 & p.R1685* & d & Recessive & (7) \\
\hline Chesler AT, et al 2016 & NM_022068.3:c.4723C>T & 32 & p.R1575* & d & Recessive & $(7)$ \\
\hline Okubo M, et al. 2015 & NM_022068.c.4456G>C & 30 & p.Ala1486Pro & DA5 & Dominant & (4) \\
\hline Delle Vedove A, et al 2016 & $\begin{array}{l}\text { NM_022068.3:c.3020_3030del } \\
\text { CTGAGAACTTC }\end{array}$ & 20 & p.Pro1007Leufs & c & Recessive & (3) \\
\hline Delle Vedove A, et al 2016 & NM_022068.c.3019_3029del & 20 & p.Pro1007Leufs*3 & c & Recessive & (3) \\
\hline McMillin MJ, et al 2014 & NM_022068.3:c.2993T>C & 20 & p.Met998Thr & DA5 & De novo & $(5)$ \\
\hline Mahmud AA, et al 2017 & NM_022068.3:c.2708C>G & 18 & p.Ser903Ter & e & Recessive & (8) \\
\hline Coste B, et al 2013 & NM_022068.3:c.2404A>T & 17 & p.Ile802Phe & DA5 & Dominant & (6) \\
\hline McMillin MJ, et al 2014 & NM_022068.3:c.2134A>G & 15 & p.Met712Val & DA5 & Dominant/de novo & (5) \\
\hline Delle Vedove A, et al 2016 & $\begin{array}{l}\text { NM_022068.3:c.1550_1552 } \\
\text { delGCTinsCGAA }\end{array}$ & 13 & p.Ser517Thrfs & c & Recessive & (3) \\
\hline Haliloglu G, et al 2017 & NM_022068, c.1384C>T & 9 & p.R462* & f & Recessive & $(1)$ \\
\hline Delle Vedove A, et al 2016 & NM_022068, c.493-?_917+del & 6,7 & NMD & c & & (3) \\
\hline
\end{tabular}

${ }^{a}$ This mutation was reported by the Institute for Human Genetics, Uniklinik RWTH Aachen (Germany) in ClinVar database (https://www. ncbi.nlm.nih.gov/clinvar/). ${ }^{b}$ Clinical phenotypes included arthrogryposis, distal, with impaired proprioception and touch. ${ }^{\mathrm{c}} \mathrm{Clinical}$ phenotypes included arthrogryposis, spontaneously resolving respiratory insufficiency at birth, muscular atrophy predominantly of the distal lower limbs, scoliosis, and mild distal sensory involvement. ${ }^{\mathrm{d} C l i n i c a l}$ phenotypes included congenital hip dysplasia, finger contractures, foot deformities, hypotonia and early severe progressive scoliosis. ${ }^{\mathrm{e}} \mathrm{Clinical}$ phenotypes included short stature, scoliosis, gross motor impairment and a progressive form of contractures involving the distal joints with impaired proprioception and touch. ${ }^{\mathrm{f}}$ Clinical phenotypes included hypotonia, distal laxity, contractures, feeding difficulties at birth. NMD, nonsense-mediated decay of PIEZO2 transcripts; PIEZO2, Piezo type mechanosensitive ion channel component 2; DA3; distal arthrogryposis 3; GS, Gordon syndrome; MWS, Marden-Walker syndrome.

\section{Discussion}

Proprioception is the perception of body and limb position mediated by proprioceptors, namely innervate muscle spindles and Golgi tendon organs, two types of mechanoreceptor in skeletal muscles. Mechanotransduction is important for sensory perception and embryonic development of organs and tissues $(9,10)$. PIEZO2 is a highly conserved non-selective 
Table II. Conservation of mutant sites of PIEZO2 in different species.

\begin{tabular}{|c|c|c|c|c|c|c|}
\hline \multirow[b]{2}{*}{ Mutant site } & \multicolumn{6}{|c|}{ Amino acid in different species } \\
\hline & $\begin{array}{l}\text { Homo } \\
\text { sapiens }\end{array}$ & $\begin{array}{l}\text { Monopterus } \\
\text { albus }\end{array}$ & $\begin{array}{c}\text { Mus } \\
\text { musculus }\end{array}$ & $\begin{array}{c}\text { Odocoileus } \\
\text { virginianus texanus }\end{array}$ & $\begin{array}{l}\text { Pogona } \\
\text { vitticeps }\end{array}$ & $\begin{array}{c}\text { Seriola } \\
\text { dumerili }\end{array}$ \\
\hline p.Trp2746Terfs & $\mathrm{W}$ & $\mathrm{W}$ & W & $\mathrm{W}$ & W & W \\
\hline p.Ser2739Pro & $\mathrm{S}$ & $\mathrm{S}$ & $\mathrm{S}$ & $\mathrm{S}$ & $\mathrm{S}$ & $\mathrm{S}$ \\
\hline p.Tyr2737Ilefs & $\mathrm{Y}$ & $\mathrm{Y}$ & $\mathrm{Y}$ & $\mathrm{Y}$ & $\mathrm{Y}$ & $\mathrm{Y}$ \\
\hline p.Glu2727del & $\mathrm{E}$ & $\mathrm{E}$ & $\mathrm{E}$ & $\mathrm{E}$ & E & $\mathrm{E}$ \\
\hline p.Arg2718Pro & $\mathrm{R}$ & $\mathrm{R}$ & $\mathrm{R}$ & $\mathrm{R}$ & $\mathrm{R}$ & $\mathrm{R}$ \\
\hline p.Arg2718Leu & $\mathrm{R}$ & $\mathrm{R}$ & $\mathrm{R}$ & $\mathrm{R}$ & $\mathrm{R}$ & $\mathrm{R}$ \\
\hline p.Arg2686His & $\mathrm{R}$ & $\mathrm{R}$ & $\mathrm{R}$ & $\mathrm{R}$ & $\mathrm{R}$ & $\mathrm{R}$ \\
\hline p.Arg2686Cys & $\mathrm{R}$ & $\mathrm{R}$ & $\mathrm{R}$ & $\mathrm{R}$ & $\mathrm{R}$ & $\mathrm{R}$ \\
\hline p.Thr2356Met & $\mathrm{T}$ & $\mathrm{T}$ & $\mathrm{T}$ & $\mathrm{T}$ & $\mathrm{T}$ & $\mathrm{T}$ \\
\hline p.Ser2223Leu & $\mathrm{S}$ & $\mathrm{S}$ & $\mathrm{S}$ & $\mathrm{S}$ & $\mathrm{S}$ & $\mathrm{S}$ \\
\hline p.Thr2221Ile & $\mathrm{T}$ & $\mathrm{T}$ & $\mathrm{T}$ & $\mathrm{T}$ & $\mathrm{A}^{\mathrm{a}}$ & $\mathrm{T}$ \\
\hline p.Trp1965Ter & W & $\mathrm{W}$ & W & W & W & W \\
\hline p.Leu1874Argfs & $\mathrm{L}$ & $\mathrm{M}^{\mathrm{a}}$ & $\mathrm{L}$ & $\mathrm{L}$ & $\mathrm{L}$ & $\mathrm{M}^{\mathrm{a}}$ \\
\hline p.Arg 1685Pro & $\mathrm{R}$ & $\mathrm{R}$ & $\mathrm{R}$ & $\mathrm{R}$ & $\mathrm{R}$ & $\mathrm{R}$ \\
\hline p.Arg1685Ter & $\mathrm{R}$ & $\mathrm{R}$ & $\mathrm{R}$ & $\mathrm{R}$ & $\mathrm{R}$ & $\mathrm{R}$ \\
\hline p.Arg 1575Ter & $\mathrm{R}$ & $\mathrm{R}$ & $\mathrm{R}$ & $\mathrm{R}$ & $\mathrm{R}$ & $\mathrm{K}^{\mathrm{a}}$ \\
\hline p.Ala1486Pro & A & $\mathrm{V}^{\mathrm{a}}$ & A & A & $\mathrm{A}$ & $\mathrm{V}^{\mathrm{a}}$ \\
\hline p.Pro1007Leufs & $\mathrm{P}$ & $\mathrm{P}$ & $\mathrm{P}$ & $\mathrm{P}$ & $\mathrm{P}$ & $\mathrm{P}$ \\
\hline p.Met998Thr & $\mathrm{M}$ & M & $\mathrm{M}$ & $\mathrm{M}$ & M & $\mathrm{M}$ \\
\hline p.Ser903Ter & $\mathrm{S}$ & $\mathrm{S}$ & $\mathrm{S}$ & $\mathrm{S}$ & $\mathrm{S}$ & $\mathrm{S}$ \\
\hline p.Ile802Phe & I & $\mathrm{V}^{\mathrm{a}}$ & I & I & I & I \\
\hline p.Met712Val & M & M & $\mathrm{M}$ & M & M & M \\
\hline p.Ser517Thrfs & $\mathrm{S}$ & $\mathrm{S}$ & $\mathrm{S}$ & $\mathrm{S}$ & $\mathrm{S}$ & $\mathrm{S}$ \\
\hline p.R462* & $\mathrm{R}^{\mathrm{a}}$ & $\mathrm{K}$ & $\mathrm{K}$ & $\mathrm{K}$ & $\mathrm{K}$ & K \\
\hline
\end{tabular}

${ }^{\mathrm{a} D e v i a t i o n ~ f r o m ~ e v o l u t i o n a r i l y ~ c o n s e r v e d ~ s e q u e n c e . ~}$

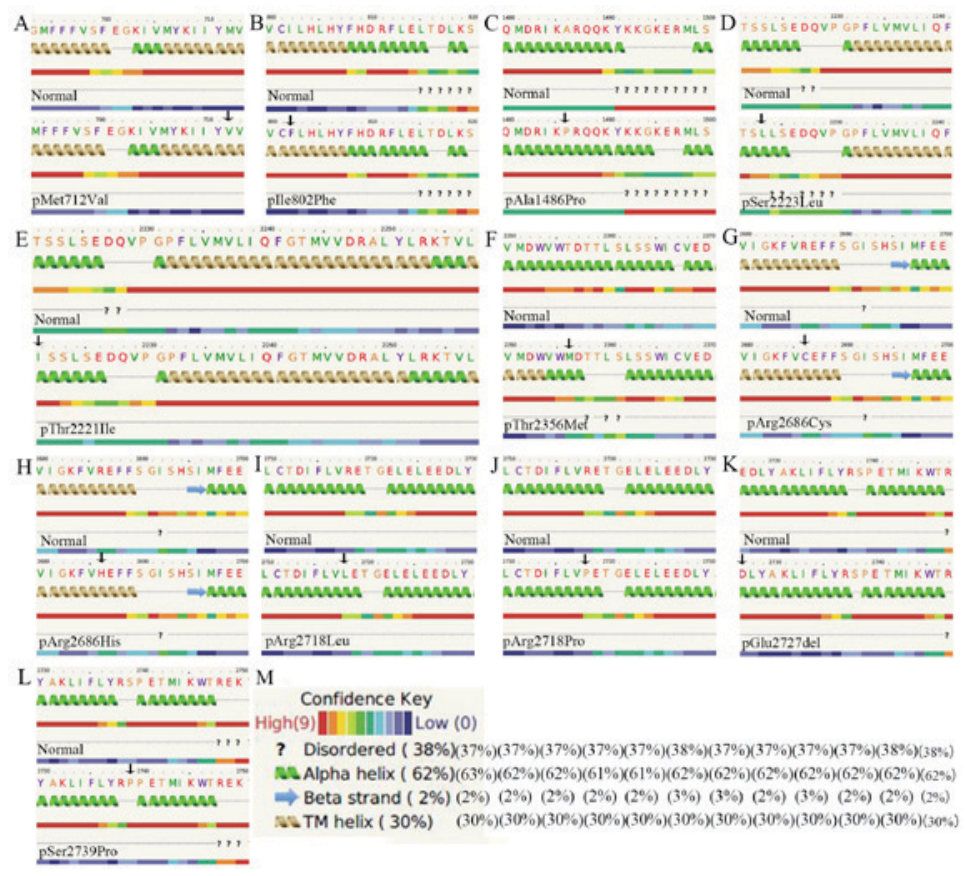

Figure 1. Prediction of the secondary structure of the human Piezo type mechanosensitive ion channel component 2 protein through the Phyre 2 online database. (A-L) Secondary structure of mutant sites of PIEZO2 protein indicated that the (C) p.Ala1486Pro, (E) p.Thr2221Ile and (K) p.Glu2727del mutations cause a modified $\alpha$ helix structure. (F) The p.Thr2356Met mutation is expected to modify the $\alpha$ helix and the TM helix structure. (M) Confidence key for the predicted structures and their pathogenicity. Arrows indicate the mutant sites. TM, transmembrane. 
pMet712Val plle802Phe pAla1486Pro pThr2221Ile

\begin{tabular}{|c|c|c|c|c|c|c|c|c|}
\hline SEQ & M & $\mathrm{V}$ & I & $\mathrm{F}$ & A & P & $\mathrm{T}$ & I \\
\hline SS3 & $\mathrm{H}$ & $\mathrm{H}$ & $\mathrm{H}$ & $\mathrm{H}$ & $\mathrm{H}$ & $\mathrm{H}$ & $\mathrm{H}$ & $\mathrm{H}$ \\
\hline SS8 & $\mathrm{H}$ & $\mathrm{H}$ & $\mathrm{H}$ & $\mathrm{H}$ & $\mathrm{H}$ & $\mathrm{H}$ & $\mathrm{H}$ & $\mathrm{H}$ \\
\hline $\mathrm{ACC}$ & E & $\mathrm{E}$ & B & B & E & E & $\mathrm{E}$ & M \\
\hline DISO & . & . & . & . & $\cdot$ & . & $\cdot$ & $\cdot$ \\
\hline & pSer22 & $223 \mathrm{Le}$ & pThr & 2356 & pArg & 2686 & pArg & g2718Le \\
\hline SEQ & $\mathrm{S}$ & $\mathrm{L}$ & $\mathrm{T}$ & $\mathrm{M}$ & $\mathrm{R}$ & C & $\mathrm{R}$ & $\mathrm{H}$ \\
\hline SS3 & $\mathrm{H}$ & $\mathrm{H}$ & C & C & $\mathrm{H}$ & $\mathrm{H}$ & $\mathrm{H}$ & $\mathrm{H}$ \\
\hline SS8 & $\mathrm{H}$ & $\mathrm{H}$ & $\mathrm{T}$ & $\mathrm{T}$ & $\mathrm{H}$ & $\mathrm{H}$ & $\mathrm{H}$ & $\mathrm{H}$ \\
\hline $\mathrm{ACC}$ & M & M & B & B & M & $\mathrm{M}$ & M & M \\
\hline DISO & $\cdot$ & . & . & . & $\cdot$ & . & $\cdot$ & $\cdot$ \\
\hline
\end{tabular}

pArg2718Leu pArg2718Pro pGlu2727del pSer2739Pro

$\begin{array}{lllllllll}\text { SEQ } & \text { R } & \text { L } & \text { R } & \text { P } & \text { E } & \text { D } & \text { P } & \text { P } \\ \text { SS3 } & \text { H } & \text { H } & \text { H } & \text { C } & \text { H } & \text { H } & \text { C } & \text { C } \\ \text { SS8 } & \text { H } & \text { H } & \text { H } & \text { L } & \text { H } & \text { H } & \text { L } & \text { L } \\ \text { ACC } & \text { M } & \text { B } & \text { M } & \text { B } & \text { M } & \text { M } & \text { M } & \text { M } \\ \text { DISO } & \text {. } & \text {. } & \text {. } & \text {. } & \text {. } & \text {. } & \text {. } & \text {. }\end{array}$

Figure 2. Prediction of the structural properties of human PIEZO2 protein through the RaptorX online database. In each pair of columns, the native site of the PIEZO2 protein is stated on the left and the corresponding mutant site on the right. The capital letters in the code correspond to the amino acids. Code in the different rows: SS3 (H, $\alpha$-helix; E, $\beta$-sheet; C, coil); SS8 [G, 3-helix; I, 5 -helix ( $\pi$-helix); $\mathrm{E}$, extended strand in $\beta$-ladder; $\mathrm{B}$, isolated $\beta$-bridge; $\mathrm{T}$, hydrogen-bonded turn; $\mathrm{S}$, hydrogen-bonded bend; L, hydrogen-bonded loop]; ACC [B, buried (ACC <10\%); M, Medium, 10\%<ACC $<40 \%$; E, Exposed, ACC $>40 \%]$. SEQ, sequence; SS3, 3-state secondary structure; ACC, solvent accessibility; DISO, disorder (prediction is based on the cutoff value at 0.25 ); PIEZO2, Piezo type mechanosensitive ion channel component 2.

and mechanosensitive cation channel (25). In line with this, the results of the present study demonstrated that these reported mutation sites are highly conserved. PIEZO2 mutations have been reported to be linked with arthrogryposis, including GS/DA3, DA5, MWS and other associated diseases $(2,3,5,6)$. However, the clinical manifestations of the PIEZO2-associated diseases display a great variation. Heterozygous gain-of-function mutations in PIEZO2 are of autosomal-dominant inheritance and contribute to GS/DA3, DA5 and MWS (6). In addition, numerous recessively inherited PIEZO2-associated diseases included arthrogryposis and other symptoms that overlap with GS/DA3, DA5 and MWS, but which may not be diagnosed as GS/DA3, DA5 or MWS due to certain distinct clinical manifestations $(1,3)$. Dominant gain-of-function mutations are mainly localized at the C-terminal end of PIEZO2. Recessive loss-of-function mutations are distributed across the PIEZO2 protein but mainly in the $\mathrm{N}$-terminal region, and do not map to any hotspots as is the case for dominant gain-of-function mutations. The results of the present study also confirm this.

A previous study indicated that gain-of-function mutations in PIEZO2 lead to a deceleration of PIZO2 channel inactivation and/or faster recovery from inactivation, resulting in increased channel activity (6). This may be due to these mutations being mainly located in the last several exons of PIEZO2 and thus, transcripts carrying these mutations are expected to escape nonsense-mediated transcript decay or premature termination codons, while mainly influencing the protein structure and function. By contrast, loss-of-function mutations may result in

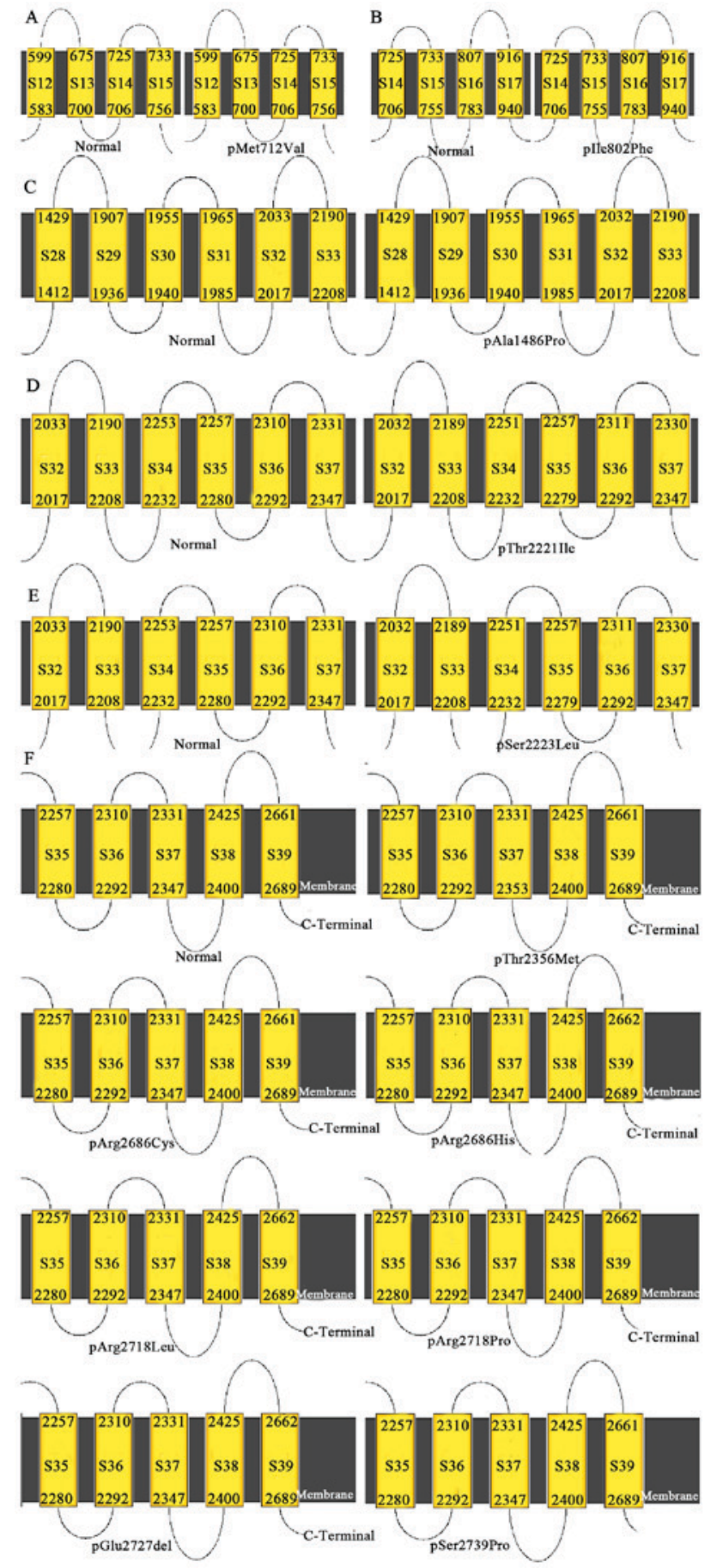

Figure 3. Prediction of transmembrane region of normal and mutant human Piezo type mechanosensitive ion channel component 2 protein through the RaptorX online database. The upper and lower numbers indicate the amino acid site. The middle number indicates the position of the transmembrane region. (A) Secondary structure of normal and p.Met712Val mutation PIEZO2 protein. (B) Secondary structure of normal and p.Ile802Phe mutation PIEZO2 protein. (C) Secondary structure of normal and p.Ala1486Pro mutation PIEZO2 protein. (D) Secondary structure of normal and p.Thr2221Ile mutation PIEZO2 protein. (E) Secondary structure of normal and p.Ser2223Leu mutation PIEZO2 protein. (F) Secondary structure of normal and p.Thr2356Met, p.Arg2686Cys, p.Arg2686His, p.Arg2718Leu, p.Arg2718Pro, p.Glu2727del or p.Ser2739Pro mutation PIEZO2 protein.

nonsense-mediated transcript decay or premature termination codons, consequently leading to a lack of PIEZO2 protein $(1,3)$. The presence of distinct clinical phenotypes between or even 
among the gain- and loss-of-function mutations of PIEZO2 linked with PIEZO2-associated diseases implies that different mutations in PIEZO2 have different effects on disease pathophysiology, which may depend on the type of mutation and the mode of inheritance.

Although the structure of PIEZO2 protein remains to be determined, the high-resolution cryo-electron microscopy structure of murine PIEZO1 has been published (26), which offers useful information for the prediction of the structure and function of PIEZO2 protein. A previous study speculated that the $\mathrm{C}$-terminal region of PIEZO2 and PIEZO1 has regulatory roles for this ion channel, which may offer certain clues regarding gain-of-function mutations (27). In the present study, the bioinformatical analysis revealed that the p.Ala1486Pro, p.Thr2221Ile and p.Glu2727del mutations modify the secondary structure of PIEZO2 protein. Furthermore, the p.Thr2221Ile, p.Arg2718Leu and p.Arg2718Pro mutations reduce the solvent accessibility of PIEZO2 protein. The p.Ala1486Pro, p.Thr2221Ile, p.Ser2223Leu, p.Thr2356Met, p.Arg2686His, p.Arg2718Leu, p.Arg2718Pro and p.Glu2727del mutations affect the transmembrane region of PIEZO2. These mutations may change the activity of PIEZO2 to enhance its function. In a previous study, similar characteristics were reported for other ion channels (28).

In summary, the results of the present study further confirm that dominant and recessive mutations were present in PIEZO2, with dominant mutations being mainly located in the $\mathrm{C}$-terminal region, whereas recessive mutations were mainly located in the N-terminal region. No overlap was present between these hotspots, and most reported mutation sites exhibited high conservation in different species, particularly in the C-terminal region. Loss-of-function mutations may result in nonsense-mediated transcript decay or premature termination codons, consequently leading to a lack of PIEZO2 protein, whereas gain-of-function mutations of PIEZO2 lead to a slowing of PIZO2 channel inactivation and/or faster recovery from inactivation, resulting in increased channel activity. The bioinformatical analysis also suggested that the p.Ala1486Pro, p.Thr2221Ile and p.Glu2727del mutations modify the secondary structure of PIEZO2 protein, while the p.Thr2221Ile, p.Arg2718Leu and p.Arg2718Pro mutations reduce the solvent accessibility of PIEZO2 protein. In addition, the p.Ala1486Pro, p.Thr2221Ile, p.Ser2223Leu, p.Thr2356Met, p.Arg2686His, p.Arg2718Leu, p.Arg2718Pro and p.Glu2727del mutations affect the transmembrane region of PIEZO2. These mutations may change the activity of PIEZO2 that may contribute to an enhanced function of PIEZO2. Variable clinical phenotypes were present between and among the gain- and loss-of-function mutations linked with PIEZO2-associated disease, which implied that different mutations in PIEZO2 have different pathophysiological effects. Of course, further functional, electrophysiological and high-resolution cryo-electron microscopy studies are required to explore the precise structure and function of PIEZO2, which may offer useful clues for the prevention and treatment of PIEZO2-associated disease.

\section{Acknowledgements}

Not applicable.

\section{Funding}

No funding received.

\section{Availability of data and materials}

The datasets used and/or analyzed during the current study are available from the corresponding author on reasonable request.

\section{Authors' contributions}

YM analyzed the data and wrote the paper. YZ and ZC analyzed the data. XH designed the study and revised the paper. The final version of the manuscript has been read and approved by all authors, and each author believes that the manuscript represents honest work.

\section{Ethical approval and consent to participate}

Not applicable.

\section{Patient consent for publication}

Not applicable.

\section{Competing interests}

The authors declare that they have no competing interests.

\section{References}

1. Haliloglu G, Becker K, Temucin C, Talim B, Küçükşahin N, Pergande M, Motameny S, Nürnberg P, Aydingoz U, Topaloglu H and Cirak S: Recessive PIEZO2 stop mutation causes distal arthrogryposis with distal muscle weakness, scoliosis and proprioception defects. J Hum Genet 62: 497-501, 2017.

2. Alisch F, Weichert A, Kalache K, Paradiso V, Longardt AC, Dame C, Hoffmann K and Horn D: Familial Gordon syndrome associated with a PIEZO2 mutation. Am J Med Genet A 173: 254-259, 2017.

3. Delle Vedove A, Storbeck M, Heller R, Hölker I, Hebbar M, Shukla A, Magnusson O,Cirak S, Girisha KM,O'Driscoll M, et al: Biallelic loss of proprioception-related PIEZO2 causes muscular atrophy with perinatal respiratory distress, arthrogryposis, and scoliosis. Am J Hum Genet 99: 1206-1216, 2016.

4. Okubo M, Fujita A, Saito Y, Komaki H, Ishiyama A, Takeshita E, Kojima E, Koichihara R, Saito T, Nakagawa E, et al: A family of distal arthrogryposis type 5 due to a novel PIEZO2 mutation. Am J Med Genet A 167A: 1100-1106, 2015.

5. McMillin MJ, Beck AE, Chong JX, Shively KM, Buckingham KJ, Gildersleeve HI, Aracena MI, Aylsworth AS, Bitoun P, Carey JC, et al: Mutations in PIEZO2 cause Gordon syndrome, Marden-Walker syndrome, and distal arthrogryposis type 5. Am J Hum Genet 94: 734-744, 2014.

6. Coste B, Houge G, Murray MF, Stitziel N, Bandell M, Giovanni MA, Philippakis A, Hoischen A, Riemer G, Steen U, et al: Gain-of-function mutations in the mechanically activated ion channel PIEZO2 cause a subtype of Distal Arthrogryposis. Proc Natl Acad Sci USA 110: 4667-4672, 2013.

7. Chesler AT, Szczot M, Bharucha-Goebel D, Čeko M, Donkervoort S, Laubacher C, Hayes LH, Alter K, Zampieri C, Stanley C, et al: The role of PIEZO2 in human mechanosensation. N Engl J Med 375: 1355-1364, 2016.

8. Mahmud AA, Nahid NA, Nassif C, Sayeed MS, Ahmed MU, Parveen M, Khalil MI, Islam MM, Nahar Z, Rypens F, et al: Loss of the proprioception and touch sensation channel PIEZO2 in siblings with a progressive form of contractures. Clin Genet 91: 470-475, 2017. 
9. Felsenthal $\mathrm{N}$ and Zelzer E: Mechanical regulation of musculoskeletal system development. Development 144: 4271-4283, 2017.

10. $\mathrm{Wu} \mathrm{J}$, Lewis AH and Grandl J: Touch, tension, and transduction - the function and regulation of piezo ion channels. Trends Biochem Sci 42: 57-71, 2017.

11. Lee W, Leddy HA, Chen Y, Lee SH, Zelenski NA, McNulty AL, Wu J, Beicker KN, Coles J, Zauscher S, et al: Synergy between Piezo1 and Piezo2 channels confers high-strain mechanosensitivity to articular cartilage. Proc Natl Acad Sci USA 111: E5114-E5122, 2014

12. Coste B, Mathur J, Schmidt M, Earley TJ, Ranade S, Petrus MJ, Dubin AE and Patapoutian A: Piezol and Piezo2 are essential components of distinct mechanically activated cation channels. Science 330: 55-60, 2010

13. Nonomura K, Woo SH, Chang RB, Gillich A, Qiu Z, Francisco AG, Ranade SS, Liberles SD and Patapoutian A: Piezo2 senses airway stretch and mediates lung inflation-induced apnoea. Nature 541: 176-181, 2017.

14. Li J, Hou B, Tumova S, Muraki K, Bruns A, Ludlow MJ, Sedo A, Hyman AJ, McKeown L, Young RS, et al: Piezol integration of vascular architecture with physiological force. Nature 515: 279-282, 2014

15. Cahalan SM, Lukacs V, Ranade SS, Chien S, Bandell M and Patapoutian A: Piezol links mechanical forces to red blood cell volume. eLife 4, 2015

16. Anderson EO, Schneider ER and Bagriantsev SN: Piezo2 in cutaneous and proprioceptive mechanotransduction in vertebrates Curr Top Membr 79: 197-217, 2017.

17. Ranade SS, Woo SH, Dubin AE, Moshourab RA, Wetzel C, Petrus M, Mathur J, Bégay V, Coste B, Mainquist J, et al: Piezo2 is the major transducer of mechanical forces for touch sensation in mice. Nature 516: 121-125, 2014.

18. Woo SH, Ranade S, Weyer AD, Dubin AE, Baba Y, Qiu Z, Petrus M, Miyamoto T, Reddy K, Lumpkin EA, et al: Piezo2 is required for Merkel-cell mechanotransduction. Nature 509: 622-626, 2014

19. Lemmers RJ, van den Boogaard ML, van der Vliet PJ, Donlin-Smith CM, Nations SP, Ruivenkamp CA, Heard P, Bakker B, Tapscott S, Cody JD, et al: Hemizygosity for SMCHD1 in facioscapulohumeral muscular dystrophy type 2: Consequences for $18 \mathrm{p}$ deletion syndrome. Hum Mutat 36 : $679-683,2015$
20. Carter E, Heard P, Hasi M, Soileau B, Sebold C, Hale DE and Cody JD: Ring 18 molecular assessment and clinical consequences. Am J Med Genet A 167A: 54-63, 2015.

21. Miller DT, Adam MP, Aradhya S, Biesecker LG, Brothman AR, Carter NP, Church DM, Crolla JA, Eichler EE, Epstein CJ, et al: Consensus statement: Chromosomal microarray is a first-tier clinical diagnostic test for individuals with developmental disabilities or congenital anomalies. Am J Hum Genet 86: 749-764, 2010

22. Wang S, Li W, Liu S and Xu J: RaptorX-Property: A web server for protein structure property prediction. Nucleic Acids Res 44: W430-W435, 2016.

23. Källberg M, Wang H, Wang S, Peng J, Wang Z, Lu H and Xu J: Template-based protein structure modeling using the RaptorX web server. Nat Protoc 7: 1511-1522, 2012.

24. Kelley LA, Mezulis S, Yates CM, Wass MN and Sternberg MJ: The Phyre 2 web portal for protein modeling, prediction and analysis. Nat Protoc 10: 845-858, 2015.

25. Vasquez V, Scherrer G and Goodman MB: Sensory biology: It takes Piezo2 to tango. Curr Biol 24: 566-R569, 2014.

26. Saotome K, Murthy SE, Kefauver JM, Whitwam T, Patapoutian A and Ward AB: Structure of the mechanically activated ion channel Piezol. Nature 554: 481-486, 2018

27. Wu J, Young M, Lewis AH, Martfeld AN, Kalmeta B and Grandl J: Inactivation of mechanically activated piezol ion channels is determined by the C-terminal extracellular domain and the inner pore helix. Cell Rep 21: 2357-2366, 2017.

28. Held K, Gruss F, Aloi VD, Janssens A, Ulens C, Voets T and Vriens J: Mutations in the voltage-sensing domain affect the alternative ion permeation pathway in the TRPM3 channel. J Physiol, 2018.

This work is licensed under a Creative Commons Attribution-NonCommercial-NoDerivatives 4.0 International (CC BY-NC-ND 4.0) License. 